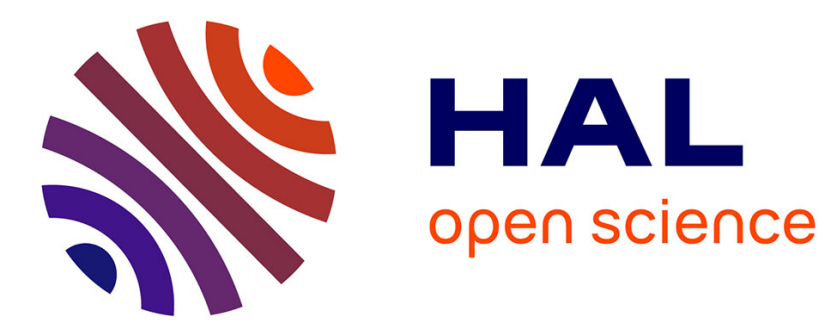

\title{
Des sentiments en acte: corps et singularité dans des relations féminines moose (Burkina Faso)
}

Virginie Vinel

\section{To cite this version:}

Virginie Vinel. Des sentiments en acte: corps et singularité dans des relations féminines moose

(Burkina Faso). CORPS : Revue Interdisciplinaire, 2012, 10.3917/corp1.010.0055 • halshs-02506505

\section{HAL Id: halshs-02506505 \\ https://shs.hal.science/halshs-02506505}

Submitted on 12 Mar 2020

HAL is a multi-disciplinary open access archive for the deposit and dissemination of scientific research documents, whether they are published or not. The documents may come from teaching and research institutions in France or abroad, or from public or private research centers.
L'archive ouverte pluridisciplinaire HAL, est destinée au dépôt et à la diffusion de documents scientifiques de niveau recherche, publiés ou non, émanant des établissements d'enseignement et de recherche français ou étrangers, des laboratoires publics ou privés. 


\title{
Des sentiments en acte : corps et singularité dans des relations féminines moose (Burkina Faso)
}

\author{
Publié dans Corps. Revue interdisciplinaire. $\mathrm{N}^{\circ} 10.2012$. pp. 55-63
}

Virginie Vinel

Laboratoire Lorrain des Sciences Sociales

Université Paul Verlaine - Metz

\begin{abstract}
Cet article propose une approche de la mise en acte des sentiments par le corps dans une société rurale africaine, les Moose du Burkina Faso. En explorant le caractère à la fois collectif et individuel des sentiments, il se conçoit également comme une contribution à une anthropologie de la singularité dans les sociétés à tendance communautaire ${ }^{1}$.
\end{abstract}

La notion de sentiment n'a pas réellement été conceptualisée contrairement aux émotions qui ont donné lieu à de nombreuses recherches en psychologie, sciences cognitives et anthropologie. J'utiliserai donc le terme pour le moment dans le sens courant de la langue française, tout en gardant à l'esprit que cette définition est nécessairement une construction historique et culturelle. Le Grand Robert de la langue française (1992 : 705) définit le sentiment comme « le fait ou la possibilité de sentir, d'éprouver, de percevoir ; et plus précisément toute tendance affective : états de plaisir ou de douleur (moral) assez stable et durable ». D'un point de vue étymologique, sentiment vient du latin, sentire : percevoir par les sens. Le sentiment comprend une dimension corporelle et psychique dans le sens où le plaisir ou le déplaisir, l'amour ou la haine, l'amitié ou l'aversion mettent en jeu des perceptions psychiques et sensorielles; de plus, le sentiment se manifeste, et c'est ce nous explorerons particulièrement, par des actions corporelles.

L'exposé se déroulera en trois temps : après un bref état des lieux des travaux anthropologiques sur les sentiments, le corps et le langage, j'aborderai la question de la place de la singularité dans les sociétés dites «traditionnelles ». La dernière partie sera consacrée à la description de deux types de relations dans lesquelles les engagements corporels actualisent des sentiments à la fois codifiés et singuliers : la relation entre sœurs et celle entre mères et filles.

\section{PETIT ETAT DE LA QUESTION EN ANTHROPOLOGIE DES SENTIMENTS}

Dans un court article de 1921, Marcel Mauss (1969) montre que l'expression des sentiments n'est pas seulement un phénomène psychologique et physiologique, mais aussi un phénomène social. S'appuyant sur l'exemple des rites funéraires dans des populations aborigènes d'Australie, il soutient que les expressions des sentiments sont collectives et obligatoires. Il note toutefois que la codification des sentiments ne nuit en rien à leur intensité. L'expression des sentiments est un langage, une symbolique, pour soi et pour les autres, conclue-t-il.

Peu de temps après, Maurice Halbwachs (1994 [1925]) affirme que les sentiments dans la famille sont appris, transmis de génération en génération: "de tels sentiments si spontanés soient-ils, suivent des chemins tracés d'avance, et qui ne dépendent point de nous, mais dont la société a pris soin d'arrêter la direction. » (ibidem : 149). L'auteur propose une différence entre le sentiment ressenti qui serait de l'ordre de l'individuel (ce qui est à questionner) et le sentiment exprimé d'ordre sociétal. Il énonce, tout comme l'ont fait les anthropologues sociaux ${ }^{2}$, le rôle des systèmes de parenté dans la construction des sentiments. 
Ces deux auteurs ont ouvert la porte d'une anthropologie des sentiments en tant qu'analyse de la construction sociale et culturelle à la fois des formes d'expression du sentiments par la parole, le chant, le corps, mais aussi des catégories de personnes pour lesquels les sentiments sont définis, les types de relations qui peuvent donner lieu ou non à des expressions publiques des sentiments. Ces auteurs ne se sont toutefois pas interrogés sur la définition que les sociétés ou les agents sociaux donnent aux sentiments, ce à quoi s'attèleront leurs successeurs. Ainsi, Eisenstadt (1956) décrit l'institutionnalisation de la relation d'amitié dans des sociétés dites traditionnelles, Paine (1969) souligne au contraire la dimension privée de l'amitié en Occident. Sinzindgre (1985) dépeint les différents types d'amitié chez les Senoufo alors que Vincent-Buffaut (1995) et Bidart (1997) respectivement d'un point de vue historique et sociologique relatent le contenu et les représentations de l'amitié en France. L'intérêt pour les sentiments, particulièrement dans la relation conjugale, s'est affirmée après les années 90 dans l'étude des sociétés « postmodernes ${ }^{3}$. L'ensemble de ces travaux nous invite à un description fine des modalités des affects et de leur expression selon le groupe observé, et à articuler davantage les actes corporels et les sentiments.

La longue tradition des recherches nord-américaines sur les émotions apportent également un cadre théorique et méthodologique à une réflexion sur sentiment et langage dans les sociétés non occidentales. Les travaux d'obédience culturalistes (Rosaldo, 1980 ; Lutz, 1988) ont montré en quoi une vision universaliste, voire sociobiologique, des émotions est à remettre en cause. M. Rosaldo (ibidem) dans une ethnographie des Ilongot, peuple de chasseur de tête aux Philippines, insiste sur la relation entre les mots et le monde. C. Lutz (1988) intensifie le travail de Rosaldo en démontrant que la base même de la notion d'émotion est éminemment culturelle. Elle le fait à partir d'une étude ethnographique dans un atoll du Pacifique sud (Ifaluk) : pour elle, les pratiques ou actes que l'on nomme "émotion» résultent d'une négociation dans un processus d'interaction entre le sens de l'événement, la morale et le droit, le contrôle des ressources. Cette perspective peut s'appliquer aux sentiments qui sont désignés comme tels dans un contexte social et une interaction. On peut les penser à la suite de Lutz, comme dynamique et en négociation. Ces auteurs nous incitent aussi à une attention aiguë aux mots et à leur situation d'énonciation.

Françoise Héritier (2004) dans un autre registre, réinvestissant la pensée de Condillac et la confrontant aux avancées contemporaines en neurobiologie explique comment perception, sensation, émotion, raison et langage sont étroitement intriqués, les premiers étant souvent à l'origine de la réflexion.

La relation entre le corps, la matière et la construction de soi a surtout été théorisée en France par le groupe Matière à Penser (Julien, Rosselin 2009) qui défendent que le sujet se construit dans un rapport dynamique, sensori-moteur, avec les objets qui l'entourent. Cette approche, qui rappelle l'ethnographie développée par W. Turner (1972) dans sa description des rituels d'affliction chez les Ndembu, nous enseigne que pour appréhender les sentiments, un regard sur les interactions corporelles dans leur dynamique et dans leur rapport aux matières, aux odeurs, aux sens mobilisés, est une piste féconde, même si difficile à réaliser du point de vue méthodologique.

Nous ne prétendons pas y aboutir dans cet exposé, mais nous tenterons de comprendre les sentiments dans leur manifestation en acte, par le corps, les gestes, les paroles, dans les interactions, notamment quotidiennes.

\section{LA SINGULARITE DANS LES SOCIETES DITES « TRADITIONNELLES »}

A la rencontre avec cet intérêt pour la vie affective s'est greffé un questionnement sur la place de l'individu et de la singularité dans les sociétés dites «traditionnelles", notamment dans le cadre de recherches de terrain au Burkina Faso. Les observations ont 
été réalisées entre 1991 et 1998 dans un quartier rural moose-sikoomse de la province du Boulkiemdé, dans un bourg de cette province, et dans la capitale, Ouagadougou (Vinel 2005). L'un des objectifs était de mettre en évidence l'agency des femmes dans un contexte social très contraignant, patrilinéaire, patriarcal, où le mariage arrangé et la polygynie prévalaient ${ }^{4}$.

Cette approche se rapporte à la question du grand partage entre les sociétés occidentales industrielles et les sociétés non européennes. Bien que remis en cause par certains anthropologues (Pouillon 1975 ; Goody 1979), la séparation entre les sociétés dites industrialisées et les sociétés dites traditionnelles n'a pas réellement été repensée du point de vue de la place de individus. La notion d'individu, en tant qu' « être moral indépendant autonome, valeur essentielle de l'idéologie individualiste des sociétés industrialisées » est difficile d'utilisation pour les sociétés non occidentales (Dumont, 1983). Mais sa définition «faible » comme « le sujet empirique, échantillon indivisible de l'espèce humaine tel qu'on le rencontre dans toutes les sociétés » (Dumont ibidem : ) semble adaptée. On lui apposera la notion de singularité, en tant que processus qui confère au type spécifique d'un être, son existence propre et concrète, déterminée dans l'espace et dans le temps, à la fois matière (corps) et psychisme, distinctes des autres individus.

De développement récent, ce questionnement sur la singularité s'insère pourtant dans un héritage anthropologique : Mauss (1989) l'a soulevé à travers la notion de personne qui motivera plus tard des recherches sur l'héritage des prénoms ou des noms, la construction de l'identité comme une conjonction de substances corporelles, spirituelles et de destin individuel (Lévi-Strauss 1983, collectif 1981, Godelier 2004). Les anthropologues insistent pourtant assez peu sur le caractère singulier de tels amalgames de substances alors que Bastide (1981) en 1971 l'avait déjà évoqué : "La singularité résulte de la formule, qui change d'une personne à l'autre, de ses appartenances. $\rangle^{5}$ D'autres anthropologues (Singularités 1989) cherchent à mettre en évidence l'émergence de personnalités exceptionnelles dans la masse des « individus ». Les récits de vie, assez nombreux dans l'anthropologie américaine, mettent quant à eux en exergue les parcours et les ressentis individuels dans des sociétés en mutation. Les travaux contemporains sur les pays du Sud ne peuvent plus faire l'économie de l'étude des parcours individuels: ainsi, les recherches publiées par Alain Marie (1997) décrivent comment les ambitions individuelles (scolarité, entreprises, migrations) se trouvent enchâsser dans des obligations et des injonctions paradoxales liées aux tensions entre la fidélité à la parenté et la pression à réussir par soi-même. Les études sur les implications sociales du VIH-Sida sont particulièrement fécondes à ce sujet (Assoun 1999 ; Fassin 2006). J-P. Warnier (2009) argue récemment que l'intérêt individuel est autant présent dans les sociétés dites traditionnelles et que le corps et les matérialités singularisent les sujets quelle que soit la société.

L'étude des sentiments et de leurs manifestations corporelles dans les sociétés à tendance communautaire sont des objets particulièrement pertinents pour aborder la part singulière de la vie sociale dans des populations rurales à fortes contraintes normatives.

\section{DES SENTIMENTS EN ACTES}

La relecture de mes notes de terrain sur les relations entre les sœurs et les cousines, entre mère et filles permettra de mettre en évidence à la fois la codification sociale et culturelle des sentiments et de leur expression, et leur caractère singulier.

Les sœurs réelles ou classificatoires (cousines parallèles patrilatérales)

Les sœurs au sens moaga du terme, c'est-à-dire les filles de même père et mère, les filles de même père, et les cousines parallèles patrilatérales ${ }^{6}$ vivent leur enfance et leur 
jeunesse ensemble. Celles qui résident dans le même enclos familial ou dans des enclos connexes partagent le quotidien: les temps des repas (elles mangent ensemble par groupe d'âge), de travail (chercher l'eau et le bois), de loisir. Jusqu'à leur mariage qui acte leur départ de l'espace familial et donc la séparation physique avec leurs sœurs, mais aussi mères, pères et frères, les sœurs de même groupe d'âge ont des modes d'expression de leur attachement visible dans les espaces publiques et privés : se promener en se tenant la main, dormir ensemble, se tresser.

Ainsi, une modalité de la relation affective est de « se suivre » (tũ taaba) surtout dans les lieux de loisir tel que le marché (giligi raaga) en se tenant la main dans ce moment privilégié pour passer du temps ensemble, exercer des complicités et partager du plaisir, des nourritures. Les jeunes filles se font voir, saluent les parents, parentes et alliés, plaisantent avec les garçons, eux-mêmes par petits groupes d'amis ou de parents liés par l'affection.

Les séances de tressage représentent aussi des temps forts d'échanges affectifs entre sœurs qui s'aiment. Ce sont des actes corporels à plusieurs dimensions : deux jeunes filles, assises à terre, coiffent une autre. Les gestes sûrs, elles s'affairent autour de la tête, parfois posé sur les genoux de la coiffeuse, enroulant, serrant, coupant. Elles sont entourées des autres jeunes filles, étendues sur les nattes au sol, qui bavardent et rient lorsque la coiffée grimace de douleur. Il s'agit aussi de rendre belles ses pairs et de construire entre soi des corps de jeunes filles séduisantes. L'assise au sol renforce la proximité des corps et la diffusion des affects.

Ces modes corporels d'expression de l'affection sont réservés aux individus de même sexe. On les retrouve pour les jeunes hommes, excepté le coiffage. Ainsi, l'expression de l'affection est codifiée, mais le choix individuel joue un rôle important: j'ai pu observer que toutes les cousines parallèles du quartier n'exerçaient pas ces modes de relations. Les jeunes filles me disaient simplement : «J'aime (nonga) ${ }^{7}$ beaucoup la/les jeunes filles de cette cour », signifiant ainsi qu'elle étaient liées davantage à ces sœurs là qu'à d'autres.

Le caractère choisi des relations entre cousines parallèles trouvent une extension dans la relation d'amitié. Un exemple l'illustre : Laale et Sore, deux sœurs classificatoires très intimes, ont trois amies (elles-mêmes sœurs classificatoires) vivant dans un autre quartier-lignage. Laale raconte qu'elle est allée les voir et leur a dit : "Si nous allons ensemble, ce sera bien. » (T'ed sã tũ taaba, yaa soma). Les jeunes filles ont accepté et l'amitié était nouée. Elles sont ainsi devenues cinq bonnes amies (zorãmba), ont acheté des pagnes de la même couleur et fait coudre un habit complet identique qu'elles portent les jours de fêtes. Elles se promènent main dans la main au marché et se font des cadeaux. Là encore, corps et objets s'articulent dans la relation affective.

Dans le gros bourg voisin, le choix et l'incidence de la relation d'affection entre jeunes filles du même âge sont plus importants. Le cas de Yasmine l'atteste : elle se rend souvent dans la cour d'un frère classificatoire de son père car « elle aime les femmes de cette cour ». La fille de ce frère de père est à la fois son amie et sa parente (elles sont cousines parallèles patrilatérales). Elle s'aident pour préparer de la bière de mil et la vendre.

A Ouagadougou, j'ai pu observer de véritables liens de solidarité entre sœurs, avec un resserrement de ce lien entre les sœurs germaines de même mère, et entre amies : les sœurs se réunissent chez l'une ou l'autre les dimanches ou pour les jours de fêtes : « Nous nous réunissons pour partager notre amour car nous sommes dispersées » dit une femme qui a pris en charge la fille de sa sœur pour qu'elle aille à l'école à côté de chez elle. Une autre femme a élevé et payé la scolarité pendant deux ans la fille de son amie car elle travaillait et ne pouvait s'en occuper. Il existe ainsi une continuité du lien entre sœurs et amies, qui souvent se recoupent en zone rurale. En zone urbaine ou semi- 
urbaine, l'affection entre sœurs germaines et entre amies est marquée par un engagement individuel plus net rendu possible par une plus grande liberté individuelle et des moyens économiques plus importants. La relation mère-filles s'avère plus ambivalente.

\section{Mères et filles}

La relation entre les mères et leurs filles est la plus solidaire que l'on ait observé, mais elle moins valorisée que la relation mère-fils. Leur destinée est étroitement liée : une fille qui refuserait un mariage risquerait de faire répudier sa mère; elles font aussi preuve de connivence en cachant au père les premières menstrues de la jeune fille pour retarder son mariage. Les mères protègent ainsi d'un mariage trop jeune.

La mère est la personne qui fait incorporer les techniques du corps féminines et elle en est tenue pour responsable. Cet apprentissage, long et quotidien, débute dès le jeune âge par des tâches domestiques, telles que passer le balai, porter le cadet sur le dos et une jarre sur la tête. Puis ils se complexifient autour de la préparation du repas qui demande l'acquisition des techniques de piler, moudre le sorgho et enfin, celle de faire cuire la pâte de mil, acte important autour de 13-14 ans qui est signifié par la mère à l'ensemble des membres de l'enclos familial. Ces apprentissages incluent des modelages corporels par la technique et par la pratique quotidienne conjointe, l'acquisition d'une résistance à la rudesse des tâches et d'une force physique. La jeune fille fait avec sa mère, le corps de celle-ci servant de modèle, avant de la remplacer vers 15 ans. Cette interaction faite d'injonction éducative et de fierté de part et d'autre, connaît des variations individuelles : certaines mères, plus sévères, utilisent l'insulte, la punition comme moyen de coercition contre des filles récalcitrantes, d'autres sont moins exigeantes. Le travail journalier fait de concert singularise aussi à la fois chaque jeune fille et chaque relation mère-fille.

Cette dynamique corporelle et affective se retrouve dans l'acte de dormir ensemble. Les garçons quittent la maison de leur mère dès 7-8 ans alors que les filles y restent jusqu'à leur mariage. Moyen de contrôle des mères sur les jeunes filles qui ne disposent pas de case personnelle, partager la même natte au sol pendant ces longues années se construit aussi comme un mode affectif d'être ensemble, qui se retrouve dans d'autres relations. Sur la natte maternelle, une hiérarchie s'établit entre le dernier né qui dort contre le sein de sa mère, et les aînés qui s'arrangent en chien de fusil le long du dos de la mère. Les enfants et les jeunes filles ont ainsi l'habitude de dormir les uns contre les autres, ce que les femmes reproduisent à l'âge adulte: Talato (22ans) raconte que nouvelle mariée, elle a dormi contre sa belle-mère pendant deux ans et qu'elle a refusé pendant longtemps de rejoindre sa maison personnelle où, malgré la présence d'enfants, elle se sent seule.

A l'intérieur de la case, mère et filles échangent sur la journée, et ces moments d'entre soi laissent place à une variabilité individuelle. Des mères plus permissives contrôlent moins leur fille, au risque de s'en faire accuser lors du mariage de celles-ci, des mères plus sévères dorment me dit-on " pied à pied contre leur fille » pour leur empêcher toute sortie nocturne. Mères et filles partagent leurs soucis, alors que d'autres restent silencieuses. Après le mariage, cette proximité corporelle se reproduit lors des visites des filles à leur mère, mais l'affection s'exprime surtout par le don.

Malgré cette proximité entre mères et filles, beaucoup de femmes interrogées sur la préférence qu'elles portent à l'un ou l'autre sexe de leur progéniture répondent les garçons : «Je veux des garçons. Si ta maison est abîmée, ton fils va la réparer. La fille, tu la donnes à son mari. Si son père devient vieux, le garçon reste. Il va te donner une maison. » " Mais, les filles vous aident ? interroge l'ethnologue à laquelle la femme répond d'une façon qui n'admet pas de réplique : « Les jeunes filles vont partir chez leur 
mari. » Certaines femmes reconnaissent, pourtant, l'attrait d'avoir des filles autant que des garçons et d'autres expriment l'affection qu'elles leur portent. La vieille Ponabda nous donne la clé de cette ambivalence et de cette variété des sentiments exprimés : «Les garçons sont supérieurs aux filles car les garçons ont des 'yiri' (habitation). La cour appartient à l'homme. Zuli est mon enfant mais la cour lui appartient, je suis là pour dormir, demander à dormir. " Cette ambivalence des sentiments, voire le déchirement qu'expriment ses femmes envers leurs filles qui s'éloignent et la perte de ce principal soutien, est le fruit de rapports sociaux de sexe inégaux, dans un système patrilinéaire et patrilocal dans lequel les hommes, père, mari, fils, forment le socle de l'habitat et du lignage. Mais entre le discours qui valorise les fils, et les pratiques qui voient quotidiennement mères et filles interagir corporellement, la singularité se construit entre distance et connivence, sévérité et affection.

\section{CONCLUSION}

Les sentiments, dans leur complexité et leur ambivalence, se matérialisent dans des actes corporels, des interactions quotidiennes qui sont le fruit à la fois d'une construction normative, sociale et culturelle, dans le cas présenté de rapports sociaux de sexe inégaux dans un contexte patriarcal, mais aussi une affaire d'engagement individuel. Le toucher (mains, cheveux) entre les jeunes filles, les corps à corps mèresenfants, les corps qui se répondent dans les apprentissages techniques, étayés sur la matière (le sol, les outils, les vêtements) produisent de l'individualité et de l'affection. Ainsi, l'entrée par les sentiments et leur mise en acte corporel et matérielle permet de déconstruire le grand partage entre les sociétés rurales et les zones urbaines, industrialisées, non pas en exerçant un ethnocentrisme et en déniant les spécificités culturelles, mais en observant à la fois les traits réguliers de définitions des sentiments mais aussi les actes, les pratiques, les discours individuels, c'est à dire en reconnaissant aux personnes de ces sociétés leur singularité.

\footnotetext{
${ }^{1}$ Dans lesquelles selon les fondateurs de la sociologie tels que Durkheim ou Tönnies, les objectifs communs dépassent les intérêts individuel.

${ }^{2}$ Notamment Alfred Reginald Radcliffe-Brown (1953).

${ }^{3}$ Antony Giddens, Niklas Luhmann, Ulrich et Elizabeth Beck, François de Singly, Jean-Claude Kaufman, Vincent Caradec, D. Lemieux se penchent ainsi sur le caractère inédit du sentiment amoureux contemporain.

${ }^{4}$ Depuis ces observations, la société moose a changé, y compris en zone rurale où les alliances sont de moins en moins arrangées faisant émerger de nouveaux enjeux dans les rapports sociaux de sexe (Laurent 2010). Mais ces transformations ne remettent pas en cause mon propos qui questionne la part de la singularité dans le contexte à forte contrainte sociale de l'époque étudiée.

${ }^{5}$ A l'inverse l'idée que le moi collectif est inexistant dans nos sociétés doit être retravaillé à la lumière des travaux de Bernard Vernier (1999) ou de Nicoletta Diasio (2010).

${ }^{6} \mathrm{C}$ 'est à dire dans la terminologie française : des sœurs, des demi-sœurs et des cousines.

${ }^{7}$ Le terme «nonga» aimer est équivalent au terme français: il est utilisé dans les relations parentsenfants, entre pairs, amies, parents, entre mari et épouse.
}

BIBLIOGRAPHIE

Bastide R. 1981, «Le principe d'individuation (Contribution à une philosophie africaine) » dans Collectif, La notion de personne en Afrique Noire, Paris, CNRS, pp. 33-43.

Bidart C. 1997, L'amitié. Un lien social, Paris, La Découverte.

Collectif, 1981, La notion de personne en Afrique noire, Paris, CNRS. 
Diasio N. 2010, «Faire corps, ou comment faire du collectif en singularisant. L'exemple des ressemblances familiales », dans Anthropologica, 52, 2: 323-336.

Dumont L. 1983, Essais sur l'individualisme, Paris, Seuil.

Eisenstadt S.N. 1956, "Ritualized personal relations" dans Man, 96 : 90-95.

Fassin D. 2006, Quand les corps se souviennent. Expérience et politiques du sida en Afrique du sud, La Découverte, Paris.

Godelier M. 2004, Les métamorphoses de la parenté, Paris, Fayard.

Goody J. 1979, La raison graphique. La domestication de la pensée sauvage, Paris, Minuit.

Halbwachs M. 1994 (1925), Les cadres sociaux de la mémoire, Paris, Albin Michel.

Héritier F. 2004, «Présentation» dans Héritier F. \& Xanthakou M., Corps et affects, Paris, Odile Jacob : 7-25.

Julien M.-P . \& Rosselin C. 2009, Le sujet contre les objets...tout contre. Ethnographies de cultures matérielles, Paris, CTHS.

Lallemand S. 1977, Une famille mossi. Recherches Voltaïques, 17, Paris-Ouagadougou, CNRS-CVRS.

Le Grand Robert de la langue française, 1992, Paris, Le Robert.

Lévi-Strauss C. 1983, L'identité, Paris, PUF.

Lutz C. 1988, Unnatural Emotion : everyday sentiments on a Micronesian atoll and their change to western theory, Chicago, University Chicago Press.

Mauss M., 1969 [1921], «L'expression obligatoire des sentiments » dans Essais de sociologie, Paris, Minuit, pp. 81-88.

Mauss M. 1989 [1938], «Une catégorie de l'esprit humain : la notion de personne celle de 'moi' » dans Sociologie et anthropologie, Paris, PUF, pp. 333-362.

Marie A. 1997, L'Afrique des individus, Paris, Karthala.

Paine R. 1969, "In Search of Friendschip : an Exploratory analysis in Middle-class Culture » dans Man, IV, $4: 505-524$.

Pouillon J. 1975, «Tradition : transmission ou reconstruction» dans Fétiches sans fétichisme, Paris, Maspero : 165-173.

Radcliffe-Brown A. R. 1953, Systèmes familiaux et matrimoniaux en Afrique, Paris, PUF

Rosaldo M. 1980, Knowledge and Passion: Ilongot Notions of Self and Social Life, Cambridge, Cambridge University Press.

Sindzingre N. 1985, «Amis, parents et alliés : les formes de l'amitié chez les Senufo (Côte-d'Ivoire) » dans Culture, Canadian Ethnology Society, 5, 2 : 69-77.

Singularités. Les voies d'émergence individuelle. Textes pour Eric de Dampierre, 1989, Paris, Plon.

Turner W. 1972, Les tambours d'affliction. Analyse des rituels chez les Ndembu de Zambie, Gallimard.

Vincent-Buffaut A. 1995, L'exercice de l'amitié. Pour une histoire des pratiques amicales aux 18 è et 19 è siècles, Paris, Seuil.

Vernier B. 1999, Le visage et le nom, Paris, PUF.

Vinel 2005, Des femmes et des lignages. Ethnologie des relations féminines au Burkina Faso (Sikoomse, Burkina Faso), Paris, L'Harmattan. 